\title{
Hough-transform based algorithm for the automatic invariant recognition of rectangular chocolates. Detection of defective pieces.
}

\author{
Recepción: Agosto de $2006 /$ Aceptación: Noviembre de 2006
}

(1) Javier Montenegro Joo

\section{ABSTRACT}

The Hough Transform has been used to develop an algorithm for rotation, translation and size-scaling invariant pattern recognition of polygonal objects. In this research the developed system has been successfully applied to the automatic invariant recognition of rectangular chocolates, including identification of defective samples. Since the entire performance of the resulting system is autonomous, the results may be applied to automatic quality control applications in the industry.

Key words: Artificial intelligence, cybernetic vision, pattern recognition, invariant shape classification, Polar Hough transform.

Algoritmo basado en la

Transformada de Hough, para el RECONOCIMIENTO INVARIANTE DE CHOCOLATES RECTANGULARES. Detección de PIEZAS falladas. RESUMEN

La Transformada de Hough (TH) ha sido usada para crear un algoritmo de reconocimiento invariante (rotación, traslación y cambio de escala) de objetos poligonales. En esta investigación, el sistema desarrollado ha sido exitosamente aplicado al reconocimiento automático invariante de chocolates rectangulares, incluyendo la identificación de piezas falladas. Como la ejecución total del sistema resultante es totalmente autónoma, los resultados pueden ser aplicados al control automático de calidad en aplicaciones industriales.

Palabras Clave: Inteligencia artificial, visión cibernética, reconocimiento del modelo, clasificación invariante de formas, Transformada Polar de Hough.
I N T ROD U C T I O N

The Hough transform [1,2] HT, is a distortion tolerant technique that maps image-space points into curves in a parameter space (also called Accumulator space). In this work the Polar, also known as Normal HT is used, this maps image-space points into a parametric Accumulator Space by means of the equation

$$
\rho=x \operatorname{Cos} \theta+y \operatorname{Sin} \theta
$$

By means of this relation, any binary point $(x, y)$ in image space $X-Y$ is mapped into a sinusoidal in the accumulator space $\rho-\theta$, which is discretized in cells of coordinates $(\rho, \theta)$. The main feature of the HT is that the $\mathrm{N}$ sinusoidals corresponding to a set of $\mathrm{N}$ collinear points $\left(\mathrm{x}_{\mathrm{i}}, \mathrm{y}_{\mathrm{i}}\right)$ meet at a point $\left(\rho_{0}, \theta_{0}\right)$ in accumulator space, the values of $\rho_{0}$ and $\theta_{0}$ characterizing the original straight line in the image. Every sinusoidal passing by $(\rho, \theta)$ contributes with 1 to a counting stored in that location. The counting stored in every cell of the accumulator corresponds to the number of points that are contained in a straight line characterized by $(\rho, \theta)$ in eq. (1).

Montenegro [12] has developed a full interactive Virtual Lab that allows the user to become acquainted with the Polar Hough Transform (PHT) while visualizing it, as well as with other Digital Image Processing topics dealing with Invariant Pattern recognition.

The algorithm being reported in this paper is mainly based on the following PHT properties, which have been thoroughly exploited:

-When a straight line is displaced to a parallel position in image space, only a proportional displacement along the $\rho$-coordinate takes place in parameter space.

- When a straight line is rotated $\Omega_{0}$ degrees in image space, its associated peak in the accumulator space is shifted $\Omega_{0}$ degrees along the $\theta$ axis.

-When an object is uniformly scaled in image space, only the associated values of $\rho$ change proportionally in the accumulator space.

Two researchers have already dealt with invariant pattern recognition in Hough space, these are Krishnapuram and Casasent [3], and Sinha et al [4], however none of these two works considered object size scaling. These works use convolution in $\theta$-space to achieve the rotational registration between sample objects and templates, an additional processing is then

(1) Director de Virtual Dynamics / Virtual Labs: Science \& Engineering Director@VirtualDynamics.Org 
necessary to determine the translational correspondence.

The algorithm developed for the work being reported here has the following advantages with respect to the two methods above mentioned:

- An algorithm to solve the problem of object size scaling has been developed and it is successfully applied. This algorithm is associated to a low complexity computation and to a short computing time.

- When training the system there is no need to place the template object in any particular position, orientation or size, it is just carelessly thrown into image-space.

- The object to be recognized is rotated and translated only once in accumulator space, then a comparison with the stored templates is carried out. This represents a substantial improvement with respect to the many comparisons implied by the methods used in references 3 and 4 .

- The counting stored in the accumulator bins (cells) is used as an evidence of sample-template matching in the present work.

In this report, RTS stands for Rotation, Translation and Size-scaling

STRATEGY OF THE ALGORITHM

This researcher has designed and developed an algorithm $[10,11]$ which consists in taking the "the object" (actually its Hough space features) to a predefined "standard status" in Hough space $[9,10,11]$. In this context, "standard status" must be understood as a previously defined "object" location, orientation, and size in accumulator space $\rho-\theta$.

The algorithm is applied equally to training and recognition stages. After Hough transforming the image-space, a sequence of operations and transformations is carried out in the accumulator, so that the object is taken to the standard status, then a characteristic vector $\mathrm{T}$ or $\mathrm{S}$, depending whether template or sample, is extracted. Montenegro [5] has already addressed the use of a pre-defined standard status in Hough space along with DistanceDiscriminator Neurons to achieve invariant pattern recognition.

At recognition time, the characteristic vectors $T$ of template objects previously extracted at the training stage are compared with the vector $S$ of the sample object to be recognized.
The distance between $\mathrm{T}$ and $\mathrm{S}$ vectors gives the corresponding T-S similitude degree.

$\begin{array}{llll}\text { ACHIEVING } & \text { RTS } & \text { INVARIANCE } & \text { IN } \\ \text { PARAMETER } & \text { SPACE } & \end{array}$

During training or recognition the object is simply thrown to image-space, in no particular position (translation), orientation (rotation), or size (scaling). However, large instances of the training object should be preferred for the sake of improved accuracy.

This algorithm operates on a single object at a time thus if several ones appear simultaneously in imagespace a pre-processing is necessary to single out individual samples.

THE TRAINING PROCESS

The object is presented only once to the system and after Hough transforming it, a set of operations take place on the accumulator space so as to take the pattern to a pre-defined standard position $[7,10,11]$, in this stage a template vector $\mathrm{T}$ is created and information about the votations obtained in the characteristic $(\rho, \theta)$ is stored, this will be used as template during the recognition process. Sometimes, depending on the special geometric characteristics of the objects used as templates, two template vectors $T_{1}$ and $T_{2}$ may be needed, this just to make sure a good performance at recognition time.

THE RECOGNITION PROCESS

When the system is on recognition mode, the same steps carried-out at the training process must be followed for the sample to be recognized. For every incognito sample a sample-vector $S$ is created with the corresponding values of $(\rho, \theta)$, and associated votations.

In order to compare sample with template, the Euclidean distance between the two T-S pairs is computed.

Advanced details of the training and recognition processes may be found in refs. 7 and 11 .

RESULTS OF THE INVESTIGATION

The performance evaluation of the developed algorithm has been previously executed with computer- 
Javier Montenegro J. 〉>>
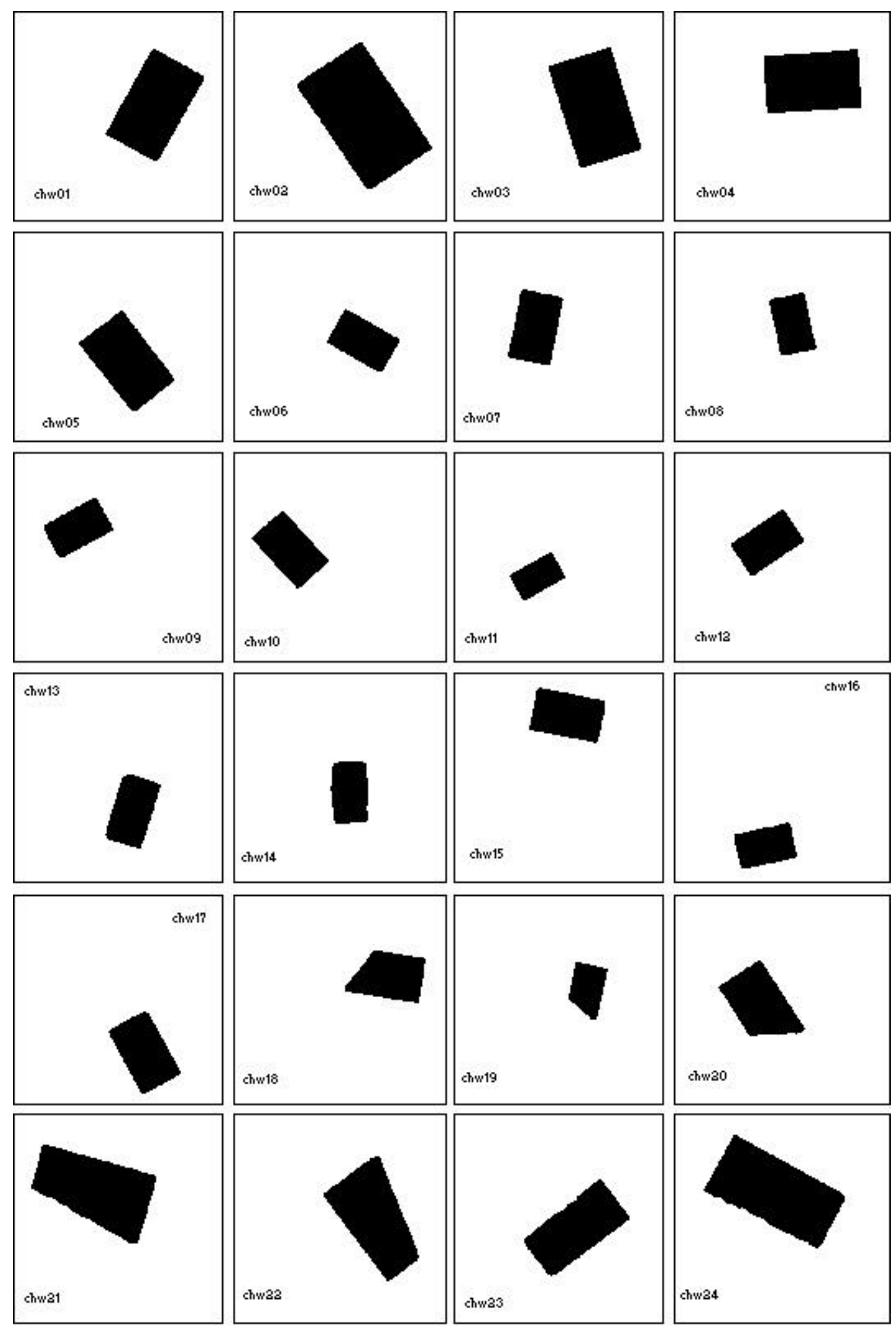

Figure 1. Chocolates from Chw01 to Chw24 used in the investigation: Flawless: Chw01 through Chw17. Flawed: Chw18 through Chw24. 
Chart 1. Template: Sample normalized Similitude Degrees.

Objects: Rectangular chocolates

Top horizontal row: templates. Left vertical column: samples

\begin{tabular}{|l|l|l|l|l|ll|l|l|l|} 
& Chw01 & Chw02 & Chw03 & Chw05 & Chw06 & Chw07 & Chw09 & Chw16 & Chw17 \\
\hline Chw & & & & & & & & & \\
\hline $\mathbf{0 1}$ & 1.000 & 0.992 & 0.987 & 0.988 & 0.990 & 0.995 & 0.995 & 0.991 & 0.979 \\
$\mathbf{0 2}$ & 0.992 & 1.000 & 0.985 & 0.988 & 0.997 & 0.992 & 0.989 & 0.984 & 0.986 \\
\hline $\mathbf{0 3}$ & 0.987 & 0.985 & 1.000 & 0.991 & 0.984 & 0.987 & 0.983 & 0.989 & 0.976 \\
\hline $\mathbf{0 4}$ & 0.984 & 0.991 & 0.979 & 0.985 & 0.992 & 0.987 & 0.982 & 0.977 & 0.992 \\
\hline $\mathbf{0 5}$ & 0.988 & 0.988 & 0.991 & 1.000 & 0.989 & 0.990 & 0.983 & 0.986 & 0.982 \\
$\mathbf{0 6}$ & 0.990 & 0.997 & 0.984 & 0.989 & 1.000 & 0.995 & 0.986 & 0.982 & 0.987 \\
\hline $\mathbf{0 7}$ & 0.995 & 0.992 & 0.987 & 0.990 & 0.995 & 1.000 & 0.990 & 0.987 & 0.982 \\
\hline $\mathbf{0 8}$ & 0.998 & 0.986 & 0.982 & 0.985 & 0.983 & 0.984 & 0.987 & 0.984 & 0.982 \\
$\mathbf{0 9}$ & 0.995 & 0.989 & 0.983 & 0.983 & 0.986 & 0.990 & 1.000 & 0.990 & 0.976 \\
\hline $\mathbf{1 0}$ & 0.980 & 0.985 & 0.972 & 0.978 & 0.986 & 0.982 & 0.978 & 0.972 & 0.985 \\
\hline $\mathbf{1 1}$ & 0.980 & 0.977 & 0.984 & 0.981 & 0.980 & 0.985 & 0.977 & 0.982 & 0.967 \\
\hline $\mathbf{1 2}$ & 0.995 & 0.987 & 0.988 & 0.988 & 0.985 & 0.990 & 0.991 & 0.991 & 0.974 \\
$\mathbf{1 3}$ & 0.975 & 0.969 & 0.975 & 0.969 & 0.967 & 0.972 & 0.973 & 0.977 & 0.955 \\
\hline $\mathbf{1 4}$ & 0.966 & 0.959 & 0.966 & 0.960 & 0.957 & 0.962 & 0.967 & 0.969 & 0.945 \\
\hline $\mathbf{1 5}$ & 0.995 & 0.992 & 0.987 & 0.990 & 0.995 & 0.992 & 0.990 & 0.987 & 0.982 \\
$\mathbf{1 6}$ & 0.991 & 0.984 & 0.989 & 0.986 & 0.982 & 0.987 & 0.990 & 1.000 & 0.972 \\
\hline $\mathbf{1 7}$ & 0.979 & 0.986 & 0.976 & 0.982 & 0.987 & 0.982 & 0.976 & 0.972 & 1.000 \\
\hline $\mathbf{1 8}$ & $0.687-1$ & $0.683-1$ & $0.681-1$ & $0.681-1$ & $0.681-1$ & $0.682-1$ & $0.689-1$ & $0.686-1$ & $0.676-1$ \\
\hline $\mathbf{1 9}$ & $0.687-1$ & $0.687-1$ & $0.482-2$ & $0.485-2$ & $0.684-1$ & $0.682-1$ & $0.689-1$ & $0.482-2$ & $0.495-2$ \\
\hline $\mathbf{2 0}$ & $0.682-1$ & $0.682-1$ & $0.674-1$ & $0.674-1$ & $0.679-1$ & $0.677-1$ & $0.684-1$ & $0.679-1$ & $0.679-1$ \\
\hline $\mathbf{2 1}$ & $0.250-3$ & $0.250-3$ & $0.250-3$ & $0.250-3$ & $0.250-3$ & $0.250-3$ & $0.250-3$ & $0.250-3$ & $0.250-3$ \\
\hline $\mathbf{2 2}$ & $0.250-3$ & $0.250-3$ & $0.250-3$ & $0.250-3$ & $0.250-3$ & $0.250-3$ & $0.250-3$ & $0.250-3$ & $0.250-3$ \\
\hline $\mathbf{2 3}$ & $0.689-1$ & $0.684-1$ & $0.694-1$ & $0.689-1$ & $0.684-1$ & $0.689-1$ & $0.689-1$ & $0.694-1$ & $0.674-1$ \\
\hline $\mathbf{2 4}$ & $0.684-1$ & $0.679-1$ & $0.689-1$ & $0.684-1$ & $0.679-1$ & $0.684-1$ & $0.684-1$ & $0.689-1$ & $0.669-1$ \\
\hline
\end{tabular}

Total samples: 24. Flawless: Chw01 through Chw17. Flawed: Chw18 through Chw24. In the case of flawed objects, the digit after the hyphen indicates the number of sample peaks out of $2 \times 2$ searching neighbourhood.

synthesized objects and with complex shape pasteboard pieces [7]. Subsequently the method has been applied to recognize biscuits [8,9]. In all cases the results show a very good performance of the algorithm.

The work reported in this paper has been performed using rectangular chocolates whose actual measures are $4.0 \times 6.7 \mathrm{cms}$, and whose photographs showing every one of them in different positions, orientations and sizes (different RTS instances), were scanned and stored in $128 \times 128$ pixel binary images, see fig. (1). In order to obtain different size samples the photographic camera was placed at varying distances from the actual objects. To make flawed chocolates, a knife was used.

Chart 1 displays the similitude degrees between the 24 chocolates and the 9 flawless pieces out of them that were carelessly chosen as templates. The samples are identified as Chwnn, where "Ch" stands for "Chocolate", "w" for the commercial brand, and "nn" is a number that runs from 01 through 24 . Pieces from Chw01 through Chw17 are flawless, whereas pieces from Chw18 through Chw24 are flawed ones.

Since the developed system is prepared to report a null similitude degree for samples with a number of sides different to that of the template, the flawed objects used in this research have all the same number of sides as that of the template, in this way the system is forced to achieve a more difficult and ambitious task.

It may be seen in chart 1 that as expected, when a template is also used as a sample the resulting similitude degree is 1.00 .

The same T-S similitude degree is obtained for any pair of objects one of them used as a template in a 
moment and as a sample in another moment. Compare results from pairs Chw01-Chw02, and Chw02Chw01, and so forth.

It can also be seen in chart 1 that flawless objects have rather high similitude degrees, while flawed objects have low similitude degrees, the numbers after the hyphen accompanying the flawed pieces similitude degrees stand for the number of accumulator peaks out of searching window. It can be seen that the higher the number of peaks out of searching window, the lower the similitude degree. Flawless pieces do not report accumulator peaks out of searching mask.

C O N C L U S I O N

The problem of rotation, translation and size-scaling (RTS) invariant pattern recognition of polygonal objects has been dealt with and an algorithm based on the fundamental properties of the polar HT has been developed and successfully applied to the invariant recognition of rectangular chocolates.

It is important to mention that in the algorithm that has been designed, the objects are taken to a predefined standard status through a set of translation, rotation, and size-scaling transformations which take place just once and exclusively in Hough space, in this way a considerable computing time is saved and the algorithm computational complexity is low.

When dealing with rectangular chocolates the algorithm successfully identifies flawless pieces, yielding a good similitude degree between template pattern and samples. The system also discriminates against flawed pieces.

The developed algorithm was initially tested with computer-synthesized objects, next with samples made with pasteboard, this was followed by its successful application to the recognition of rectangular biscuits and now it has been applied to the recognition of chocolates including discrimination against flawed pieces. These results suggest that this algorithm may be profitably applied to industrial automatic quality control of products, maybe for objects being transported on a conveyor belt.

\section{A C K N O W E D G E M E T}

This work would not have been possible without the deep and decisive influence of Prof. Luciano da Fontoura Costa, from The Cybernetic Vision Research Group, Sao Paulo University, (USP Sao Carlos Brasil), who introduced the author to the field of Computer
Vision, specifically Pattern Recognition. The author expresses his sincere and special gratitude to Prof. da Fontoura Costa for all the time, dedication and very specially for the transfer of knowledge.

BIBLIOGRAPHICAL

REFERENCES

1. Illingworth J. and Kittler J. (1998). A survey of the Hough transform. CVGIP, vol , 44, pags 87-116.

2. Duda R.O. and Hart P.E. (1972). Use of the Hough transform to detect lines and curves in pictures. Communications of the ACM, Vol 15, No 1, 1115, January.

3. Krishnapuram R. and Casasent D. (1987). Hough space transformations for discrimination and distortion estimation. CVGIP, vol 38, pags. 299316.

4. Sinha P.K., Chen F.Y., Horne R.E.N. (1993). Recognition and location of shapes in the Hough pattern space. IEE Elect. Div. Colloq. on Hough transform, 1993/106. Savoy place, London, May.

5. Montenegro J.J. - Da Fontoura Costa L. - Köberle R. (1993). Geometric-Transformation-Invariant pattern recognition with Hough transforms and Distance-Discriminator Neural Networks. Workshop sobre Computacaõ de alto desempenho para processamento de sinais. São Carlos, SP, Brasil.

6. Gerig G. and Klein F. (1986). Fast contour identification through efficient Hough transform and simplified interpretation strategy. IJCPR-8, Paris, 498-500.

7. Montenegro J. J. (1994). Doctoral thesis research under the direction of Professor Luciano da Fontoura Costa (Cybernetic Vision Research Group, USP-SC, Brasil.

8. Montenegro J. J. (2002). Invariant Recognition of rectangular biscuits through an algorithm operating exclusively in Hough space. Flawed pieces detection. Revista de Investigacion de Fisica, Vol 5 , No 1,2 .

9. Montenegro J. J. (1995). Inteligencia Artificial: Reconocimiento de objetos en visión computarizada. Seminario Software Científico, CONCYTEC: National Sci. \& Tech Council, Lima Perú.

10. Montenegro J. J. (2004). Invariant pattern recognition executed exclusively in the Hough 
〉> Hough-transform based algorithm for the automatic invariant recognition of rectangular chocolates. Detection of defective pieces.

Space. Revista Electronica-UNMSM, No 13, Aug. 2004

11. Montenegro J. J. (1998). A Polar-Hough-Transform Based Algorithm for the Translation, Orientation and Size-Scale Invariant Pattern Recognition of
Polygonal Objects. UMI Dissertations Microform LD03769. Oct. 1998

12. Montenegro J. J. Imagery, Digital Image Processing Virtual Laboratory. Virtual Dynamics / Virtual Labs: Science \& Engineering. 